\title{
Global transcriptional response of Aspergillus niger in the process of glucoamylase fermentation
}

\author{
Yu-fei Sui ${ }^{1+}{ }^{\oplus}$, Li-ming Ouyang ${ }^{1 \dagger}$, Ju Chu' ${ }^{1}$, Wei-qiang Cao ${ }^{1}$, Li-feng liang ${ }^{2}$, Ying-ping Zhuang ${ }^{1 *}$, Shu Cheng ${ }^{2^{*}}$, \\ Henk Norrman ${ }^{3}$, Si-liang Zhang ${ }^{1}$ and Geng-yun zhang ${ }^{2}$
}

\begin{abstract}
Background: In the phase of oxygen limitation during Aspergillus niger fermentation, the cell growth decreased, while the yield of glucoamylase increased continuously and significantly. Explanation on the changes of transcriptome profile during this process may improve our understanding on mechanisms of cell adaption and enzyme production in A. niger.
\end{abstract}

Results: The transcriptomic data from 4 time points in oxygen limitation process of a glucoamylase production $A$. niger strain were analyzed. Hierarchical clustering of all samples showed that the strongest transcriptional response occurred between the early and middle stage of oxygen limitation. 515 differentially expressed genes (DEGs) were identified and were clustered into 12 expression patterns. Continuously down-regulated DEGs were significantly enriched in GO terms of the ribosome, translation, and aminoacyl-tRNA biosynthesis, and continuously up-regulated DEGs were mainly involved in GO terms of fatty acid catabolism, N-acetyltransferase activity, lipase activity, and carboxylesterase activity. Pyruvate kinase and asparagine synthetase which related to the biosynthesis of the main amino acid composition of the enzyme were significantly up-regulated. Sterol-regulatory element-binding proteins (SREBP) transcription factor SrbB was one of the most up-regulated proteins, indicating its important roles in hypoxia fermentation of A. niger.

Conclusion: Comparative transcriptome data analysis of the fermentation revealed that the overall reduced biosynthesis of translation machine and fatty acid, acceleration of fatty acid catabolism, and increased synthesis of main amino acid compositions of glucoamylase are the key transcriptional changes during the oxygen limitation fermentation process of $A$. niger.

Keywords: Aspergillus niger, Glucoamylase, Transcriptome, Metabolic pathways, Oxygen limitation, Fermentation

\section{Background}

Aspergillus niger, a kind of ascomycetous filamentous fungi, is very common in the environment (Baker 2006). Under the natural growth condition, A. niger secretes a large number of enzymes with multi-functions to degrade biopolymer in the environment to obtain the

\footnotetext{
*Correspondence: ypzhuang@ecust.edu.cn; chengshu@genomics.cn

${ }^{\dagger}$ Yu-fei Sui and Li-ming Ouyang contributed equally to this work

${ }^{1}$ State Key Laboratory of Bioreactor Engineering, East China University of Science and Technology, Shanghai 200237, People's Republic of China

2 BGI-Shenzhen, Shenzhen 518083, China

Full list of author information is available at the end of the article
}

nutrition. The industrial enzymes produced by $A$. niger played a great role in the field of starch processing, fermentation, brewing, beverage production, animal feed, and paper industry. In addition, $A$. niger can also be used as a cell factory to yield homologous as well as heterologous protein, citric acid, gluconic acid, and glucoamylase, and has been widely applied in the fermentation industry due to its merits of high yield, high secretion, and high security (Pel et al. 2007). Among the diverse products yielded by $A$. niger, glucoamylase (also known as amyloglucosidase), which can hydrolyze more than $80 \%$ of the starch with high activity in general (Pavezzi et al. 2011), 
has been identified as food safety products and is widely used in food and brewing industry.

During the growth of aerobic microorganisms, oxygen is an important electron acceptor in the respiratory chain. Along with the oxidation of NADH and FADH2, electrons are transferred to oxygen through electron transfer chain, and the released energy leads to the formation of ATP, which provides energy for strain growth and metabolism (Diano et al. 2009). Hence, the oxygen availability is generally one of the most important factors in the fermentation process of aerobic microorganisms. During the fermentation of filamentous fungi, the viscosity of the medium increased with the increase of biomass (Pedersen et al. 2000), which causes the enhancement of transfer resistance and the reduction of oxygen transfer efficiency, and hence inevitably resulted in the oxygen limitation regardless of high power agitation and aeration (Diano et al. 2006). Pedersen et al. (2012) simulated actual glucoamylase industrial fermentation process for studying the regulation mechanism of physiological metabolism, and it has been observed that during the whole fermentation process, the yield of glucoamylase on substrate was the highest in oxygen limitation phase. Hence, oxygen limitation has been applied as the most effective strategy for industrial glucoamylase production at present. Some studies have revealed the complex relation between the specific productivity $\left(\mathrm{q}_{\mathrm{p}}\right)$ of glucoamylase and the specific growth rate $(\mu)$. When $\mu$ is lower than $0.1 \mathrm{~h}^{-1}$ under chemostat cultivation, $\mathrm{q}_{\mathrm{p}}$ is coupled with $\mu$. However, when the specific growth rate is higher than $0.1 \mathrm{~h}^{-1}, \mathrm{q}_{\mathrm{p}}$ will decrease, which indicates that more precursors and energy flow to cell growth (Pedersen et al. 2000, 2012). So, it was estimated that sufficient supplement of oxygen accelerates the carbon flux flowing to cell growth of $A$. niger. Moreover, oxygen limitation may repress the synthesis of biomass, allowing remained precursors and energy flows to the synthesis of glucoamylase and by-products. In order to obtain a more distinct description about cell metabolism under oxygen-limited conditions, Diano et al. (2009) explored the formation mechanism of different by-products in $A$. niger under oxygen limitation condition via chemostat culture. The results showed that the concentration of TCA cycle intermediates increased and the activity of respiratory chain decreased under low oxygen availability, resulting in the accumulation of NADH and the decrease of ATP, respectively. The excessive reduction power leads to the formation and secretion of organic acids. Although oxygen limitation is usually used for industrial production of glucoamylase, the genome-wide expression response and regulation mechanisms of $A$. niger under oxygen-limited conditions are still lacking in-depth study (Lu et al. 2015).
The data analysis at the whole transcriptome level makes researchers assess the relationship between cell phenotype and gene expression more accurately, and deepen the understanding of cellular control on metabolism (Shi et al. 2010). The increasing progress of sequencing technology has promoted the development of transcriptomics and its application in the physiology study of $A$. niger, such as explaining the efficient enzyme production mechanism (Kwon et al. 2012; Andersen et al. 2011; Jørgensen et al. 2010), the global response mechanism under different carbon sources (Jørgensen et al. 2009; Delmas et al. 2012; van Munster et al. 2014; Nitsche et al. 2012), and in the process of spore germination (Novodvorska et al. 2013; van Leeuwen et al. 2012).

At present, the reports on oxygen limitation in fermentation process of filamentous fungi are mainly about the physiological data and metabolomics to describe the parameter changes during the oxygen-limited stage (Pedersen et al. 2012; Diano et al. 2006, 2009; Lu et al. 2015), while the global response at transcriptional level is not well studied. In this paper, we analyzed the transcriptomic data from samples of different stages in the process of glucoamylase fermentation using a strand-specific RNA-Seq approach. The goals of this study were to investigate the global transcriptional response in different stages of the oxygen-limited fermentation process of glucoamylase production by $A$. niger, in order to understand the metabolic regulation mechanism at the transcriptional level better.

\section{Methods \\ Strain}

The strain A. niger DS03043 used in this paper was provided by DSM (Delft, the Netherlands). A. niger DS03043 is an industrial glucoamylase-producing strain with a high yield, which contains seven copies of the glucoamylase-coding gene gla $A$ in the genome.

\section{Culture in conical flask}

In order to obtain $A$. niger spores, the frozen spores (preserved in $50 \%$ glycerol at $-80{ }^{\circ} \mathrm{C}$ ) were inoculated on the PDA plates. The seed medium consisted of glucose $22 \mathrm{~g} / \mathrm{L}$ and corn syrup solid powder $20 \mathrm{~g} / \mathrm{L}$ and the initial $\mathrm{pH}$ of the medium was adjusted with $3 \mathrm{M} \mathrm{NaOH}$ to 6.5 before sterilization. The seed culture was carried out in $500-\mathrm{mL}$ shake flasks with band baffle, and the inoculation amount of each shake flask was $10^{7}$ spores $/ 100 \mathrm{~mL}$ media. Then, the flasks were incubated at $34{ }^{\circ} \mathrm{C}$ on a rotary shaker (250 rpm) for $24 \mathrm{~h}$.

\section{Bioreactor fed-batch fermentation}

This study adopted the synthesis medium for all fedbatch culture $(\mathrm{g} / \mathrm{kg})$, including glucose $\cdot \mathrm{H}_{2} \mathrm{O} 20, \mathrm{KH}_{2} \mathrm{PO}_{4}$ 
3, $\mathrm{NaH}_{2} \mathrm{PO}_{4} \cdot \mathrm{H}_{2} \mathrm{O}$ 1.5, $(\mathrm{NH} 4)_{2} \mathrm{SO}_{4} \quad 3, \quad \mathrm{MgSO}_{4} \cdot 7 \mathrm{H}_{2} \mathrm{O}$ 1, $\mathrm{CaCl}_{2} \cdot 2 \mathrm{H}_{2} \mathrm{O} 0.1, \mathrm{ZnCl}_{2} 0.02, \mathrm{CuSO}_{4} \cdot 5 \mathrm{H}_{2} \mathrm{O}$ 0.015, $\mathrm{CoCl}_{2} \cdot 6 \mathrm{H}_{2} \mathrm{O} \quad 0.015, \quad \mathrm{MnSO}_{4} \cdot \mathrm{H}_{2} \mathrm{O} \quad 0.04, \quad \mathrm{FeSO}_{4} \cdot 7 \mathrm{H}_{2} \mathrm{O}$ 0.3. The fed-batch culture was performed on a 5 -L bioreactor (Guoqiang Biological Engineering Equipment Technology Co., LTD, Shanghai, China) containing $3 \mathrm{~L}$ fermentation broth with online monitoring function for temperature, $\mathrm{pH}$, stirring speed, dissolved oxygen (DO), oxygen uptake rate (OUR), carbon dioxide evolution rate (CER), respiration quotient (RQ), etc. $A$. niger was cultured at $34{ }^{\circ} \mathrm{C}$ with the stirring speed of $375 \mathrm{rpm}$ and the aeration rate was set at $1 \mathrm{VVM}$ during the whole process. The $\mathrm{pH}$ of fermentation broth was controlled by ammonia $(5 \% \mathrm{~W} / \mathrm{W})$ at 4.5 . When the glucose concentration of the fermentation broth was reduced to $5 \mathrm{~g} / \mathrm{L}$, the supplement was started by adjusting the feed rate to maintain the sugar concentration of the culture at about $5 \mathrm{~g} / \mathrm{L}$. The experiments were repeated for three times under the same conditions.

\section{Sampling for RNA-Seq}

Cellular transcriptome was sampled at 4 time points in the fermentation process, viz. $16 \mathrm{~h}$ (HA) in the exponential growth phase, $24 \mathrm{~h}(\mathrm{HB})$ in the early phase of oxygen limitation, $42 \mathrm{~h}(\mathrm{HC})$ in the middle phase of oxygen limitation, and $66 \mathrm{~h}$ (HD) in the late phase of oxygen limitation. Total RNA for RNA-Seq was taken from triplicates under three parallel experiments.

\section{Enzyme essay}

One unit of enzyme activity (AGI) was defined as the amount of enzyme needed to hydrolyze soluble starch per minute to produce $1 \mathrm{~mol}$ glucose at $\mathrm{pH}=4.3,60^{\circ} \mathrm{C}$.

To determine the glucoamylase activity of culture, $2 \mathrm{~mL}$ fermentation broth was centrifuged at 10,000 rpm for $5 \mathrm{~min}$, and then the supernatant was diluted with deionized water to the appropriate concentration (10-40 AGI $\left.\cdot \mathrm{mL}^{-1}\right) .230 \mu \mathrm{L}$ p-nitrophenyl- $\beta$-D-galactopyranoside (p-NPG, $2 \mathrm{~g} / \mathrm{L}$ dissolved in $\mathrm{pH}=4.3$ acetic acid sodium acetate buffer) was preheated at $37{ }^{\circ} \mathrm{C}$ for $5 \mathrm{~min}$, and then immediately reacted with $20 \mu \mathrm{L}$ the diluted supernatant of fermentation broth at $37^{\circ} \mathrm{C}$ for $20 \mathrm{~min}$. At last, add $100 \mu \mathrm{L} 0.3 \mathrm{~mol} / \mathrm{L}$ sodium carbonate to terminate the reaction and immediately read the absorbance at $405 \mathrm{~nm}$.

Glucoamylase standard sample was diluted to a series of concentration gradients and the absorbance values were measured by microplate spectrophotometer at $405 \mathrm{~nm}$ according to the above steps. The standard curve equation was achieved as follows:

$$
\begin{aligned}
\text { Glucoamylase activity }= & \text { dilution ratio } \\
& \times \frac{O D_{405}+0.01}{0.008}\left(R^{2}>0.999\right)
\end{aligned}
$$

Using the same measuring procedure and the standard curve to calculate the glucoamylase activity.

\section{Dry weight}

The biomass of $A$. niger was indicated by the dry weight. Filter $5 \mathrm{~mL}$ fermentation broth with a dried filter paper, followed by washing for 3 times with deionized water. And then the wet biomass was transferred to the electric oven at $80{ }^{\circ} \mathrm{C}$ for $24 \mathrm{~h}$, after that the dried biomass was weighed immediately.

\section{RNA extraction}

Three biological replicates of RNA samples were frozen directly in liquid nitrogen and stored at $-80{ }^{\circ} \mathrm{C}$. The extraction and quality detection of transcriptional samples were performed by Sangon biotech (Shanghai, P.R. China) Co., Ltd.

\section{Strand-specific RNA-Seq}

Beads containing oligo (dT) were used to isolate poly $(\mathrm{A})$ mRNA from total RNA. Purified mRNA was then fragmented. Using these short fragments as templates, random hexamers were used for first-strand cDNA synthesis. The second-strand cDNA was synthesized using buffer, dNTP/dUTP mix, RNase $\mathrm{H}$, and DNA polymerase I. Short double-strand cDNA fragments were purified using a QIAquick PCR extraction kit and eluted with EB buffer for end repair, with the addition of an 'A' base, and ligated to Illumina sequencing adaptors. Then, the second-strand cDNA was excised by UNG enzyme. The fragments with expected size were purified and then amplified by PCR. The amplified library was sequenced on an Illumina $\mathrm{HiSeq}^{\mathrm{TM}} 2000$ sequencing machine. The details of the experiment were as follows: expected library size: $200 \mathrm{bp}$; read length: $90 \mathrm{nt}$; and according to the sequencing strategy: paired-end sequencing.

The clean reads were produced after the raw reads were filtered to the following criteria: remove reads with sequence adaptors; remove reads with more than $10 \%$ ' $N$ ' bases; and remove low-quality reads, which have more than $50 \%$ QA $\leq 10$ bases. All subsequent analyses were based on clean reads.

\section{Mapping reads to the reference genome and normalization of gene expression}

The reference sequence used was the genome sequence of $A$. niger strain CBS 513.88 (Genbank IDGCA_000002855.2). Clean reads were then aligned to the reference genome and no more than three mismatches were allowed in the alignment for each read.

Reads that could be uniquely mapped to a gene were used to calculate the expression level. The gene expression level was quantified using RSEM (Li and Dewey 2011). 
The formula is as follows:FPKM $=\frac{10^{6} \mathrm{C}}{N L / 10^{3}}$, in which FPKM (A) is the expression level of gene A. $C$ is counts of fragments that uniquely mapped to the gene A. $N$ is total counts of fragments that uniquely mapped to the reference genes. $L$ is the base counts of the coding region of gene A. FPKM method can eliminate the influence of gene length and difference of sequencing on the calculation of gene expression, and the calculated expression of the gene can be directly used to compare gene expression between different samples.

\section{Differentially expressed gene analysis}

We identified differentially expressed genes between paired time point samples using the EBSeq method (Ning et al. 2013) based on the following criteria: FDR $\leq 0.05$ and fold change $\geq 2$. All DEGs among the four time points were merged as DEGs union set for gene expression pattern analysis. Then, a dendrogram was created after the expression of each gene was normalized across the four time points by dividing the mean of FPKM value at each time point by the maximum of mean FPKM value for the same gene. The hierarchical clustering analysis was performed using unweighted pair group method with arithmetic mean.

\section{Functional annotation and GO and KEGG classification}

NCBI non-redundant protein database (Nr) (https:// www.ncbi.nlm.nih.gov), David Bioinformatics Resources 6.7 (DAVID) (https://david.ncifcrf.gov) (Huang et al. 2009a, b), Aspergillus genome database (AspGD) (http:// www.aspgd.org) (Cerqueira et al. 2014), and kyoto encyclopedia of genes and genomes (KEGG) (http://www. kegg.jp) (Kanehisa and Goto 2000; Kanehisa et al. 2016) were used for functional annotation of all DEGs. GOSlim in AspGD database and $R$ package ggplot2 were performed for gene ontology (GO) classification and plotting, respectively. iPath (http://pathways.embl.de) (Yamada et al. 2011), a user-friendly online software, can visualize the distribution of DEGs on different metabolic pathways in a metabolic network more intuitively.

\section{Results and discussion}

\section{Growth physiology in the process of glucoamylase fermentation of $A$. niger}

The commonly used industrial fed-batch culture was performed in this study. During the whole fermentation process, the concentration of glucose was maintained above $5 \mathrm{~g} / \mathrm{L}$, and thus eliminated the influence of carbon limitation on the metabolism of $A$. niger. The whole fed-batch fermentation process could be roughly divided into two stages according to dissolved oxygen (DO) curves, which were the stage with sufficient oxygen supply $(0-24 \mathrm{~h})$ and oxygen-limited stage (24-72 h) (Fig. 1a). During the exponential growth phase (8-24 h), along with the fast growth of cells, oxygen uptake rate (OUR) increased dramatically and DO declined rapidly (Fig. 1a), which in turn resulted in the continuous decrease of $\mu$ (Fig. 1b). With the booming of biomass (Fig. 1d), the viscosity of fermentation broth increased rapidly, which finally caused DO reaching the lowest level and indicated the entry of fermentation into the oxygen limitation period. OUR decreased rapidly after entering the oxygen limitation period and then maintained at a stable level, during which $\mu$ decreased rapidly (Fig. 1a, b). In this study, four transcriptome sampling time points were chosen according to the curve of OUR at 16, 24, 42, and $66 \mathrm{~h}$ in the process of fermentation. These time points were corresponding to the exponential growth phase (HA), the early stage of oxygen limitation (HB), the middle stage of oxygen limitation, and the late stage of oxygen limitation (HD), respectively. During 18-24 h, although $\mu$ decreased rapidly, OUR and $\mathrm{q}_{\mathrm{p}}$ still dramatically increased, which indicated the metabolic balance between the biosynthesis of glucoamylase and the growth of biomass had been changed in this phase, and more flux was redistributed to the former. From the view of glucoamylase production, this stage may be ideal to keep a high $\mathrm{q}_{\mathrm{p}}$ level and a moderate growth rate. After that, with the continuous reduction of $\mu, \mathrm{q}_{\mathrm{P}}$ decreased significantly but was not as fast as $\mu$ during 24-42 h (Fig. 1b). In the exponential growth phase $(<18 \mathrm{~h})$, the enzyme activity of glucoamylase increased gradually (Fig. 1e), and therefore, the yield of the glucoamylase on biomass decreased with the increase of $\mu$. After coming into the oxygen limitation phase, the enzyme activity accumulated rapidly (Fig. 1e), and the yield of the glucoamylase on biomass displayed a linear increase trend, which reached more than three times of that in the exponential growth phase (Fig. 1c).

\section{Overview of RNA-Seq data}

Triplicate samples of four time points in the fermentation process were collected in this study and 11 qualified RNA samples were then sequenced. Each individual sample generated approximate 0.35 billion base data (in 90-bp paired-end raw reads). Among them, clean reads accounted for $96.01-98.33 \%$ viz. about $0.33-0.34$ billion data of each sample after quality control. The high-quality reads of all the samples were mapped to the published CBS 513.88 (Genbank Accession Number: GCA_000002855.2) genome. For the transcriptome sequences of each sample, mapped percentage to the reference genome and genes were $83.22-85.04 \%$ and $61.28-62.31 \%$, respectively, of which $79.52-83.37 \%$ of reads were uniquely mapped to the reference genome, and $57.67-61.01 \%$ of reads were uniquely aligned to the reference genes (Table 1 ). The RNA-Seq data are listed in Additional file 1: Table S1. 

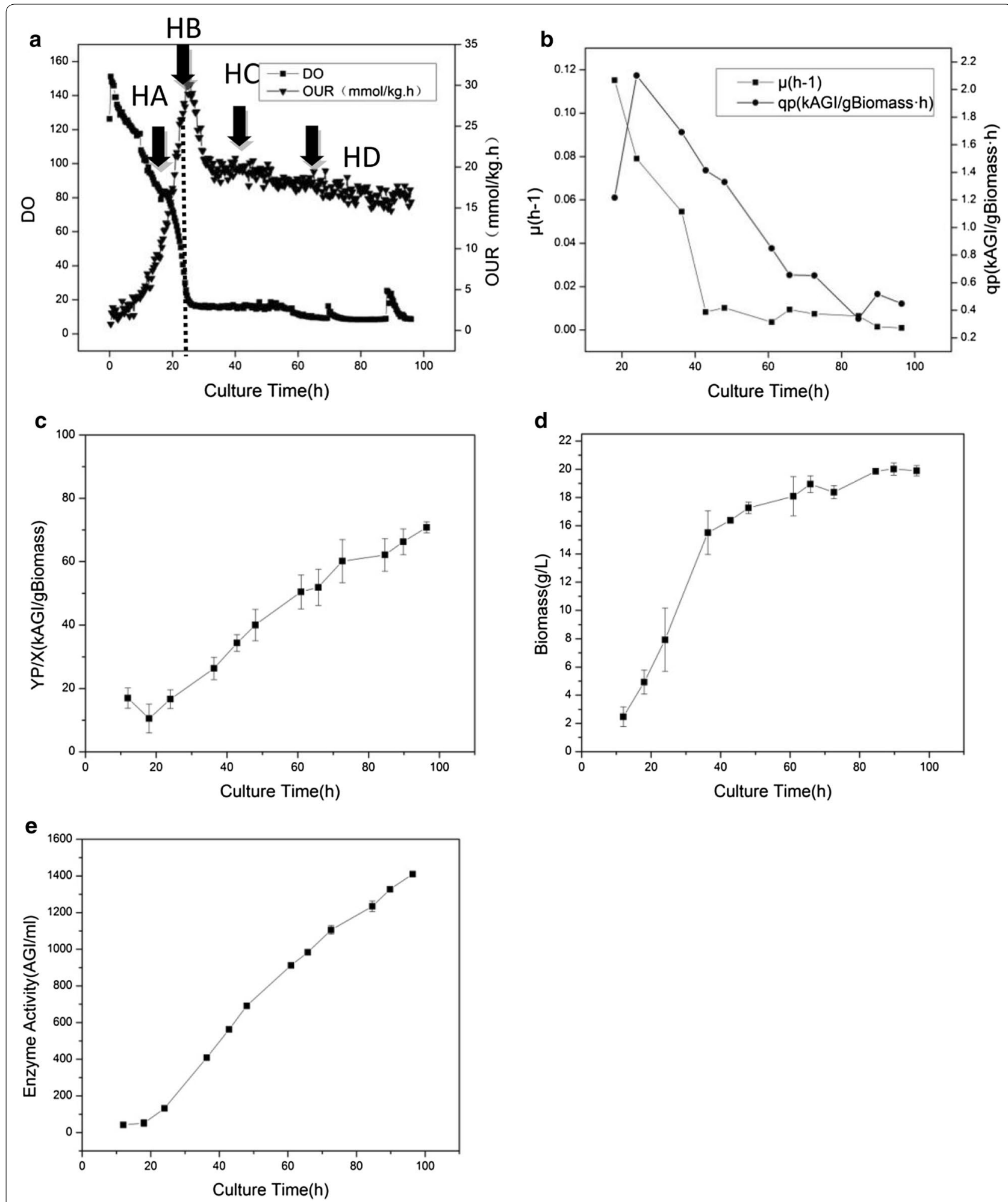

Fig. 1 Growth profiles of A. niger DS03043 during fed-batch cultivation (Arrows represents four sampling time points at 16, 24, 42, and 66 h). a Online data of DO and OUR. After the dotted line, it came into the oxygen limitation stage; $\mathbf{b}$ off-line data of $\mu$ and $\mathrm{q}_{\mathrm{p}}$; $\mathbf{c}$ the yield of glucoamylase to biomass; $\mathbf{d}$ the changes of dry weight; $\mathbf{e}$ enzyme activity of glucoamylase. All parameters were measured based on triplicate measurements 
Table 1 Sequencing and assembly statistics for the 11 transcriptome data of $A$. niger DS03043 at four time points in the process of glucoamylase fermentation

\begin{tabular}{|c|c|c|c|c|c|c|}
\hline Sample ID & $\begin{array}{l}\text { No. of clean } \\
\text { reads }\left(\times 10^{6}\right)\end{array}$ & $\begin{array}{l}\text { No. of base } \\
\text { pairs }\left(\times 10^{9}\right)\end{array}$ & $\begin{array}{l}\text { No. of mapped } \\
\text { reads (to genome) } \\
\left(\times 10^{6}\right)\end{array}$ & $\begin{array}{l}\text { Mapped } \\
\text { percentage } \\
\text { to genome (\%) }\end{array}$ & $\begin{array}{l}\text { No. of mapped } \\
\text { reads (to gene) } \\
\left(\times 10^{6}\right)\end{array}$ & $\begin{array}{l}\text { Mapped } \\
\text { percentage } \\
\text { to gene (\%) }\end{array}$ \\
\hline $\mathrm{HA}-16 \mathrm{~h}$ & 35.43 & 3.54 & 29.95 & 84.54 & 21.71 & 61.28 \\
\hline HB-24 h & 35.12 & 3.51 & 29.23 & 83.22 & 21.81 & 62.08 \\
\hline $\mathrm{HC}-42 \mathrm{~h}$ & 33.63 & 3.36 & 28.60 & 85.04 & 20.96 & 62.31 \\
\hline HD-66 h & 33.83 & 3.38 & 28.53 & 84.32 & 20.92 & 61.82 \\
\hline
\end{tabular}

\section{Functional and expression pattern analysis of DEGs}

Correlation analysis for triplicate samples by $\mathrm{R}$ package revealed that the Pearson correlation coefficient (PCC) among replicates was very high as between 96.1 and $99.6 \%$, which meant the repeatability of each replicate was good. Hierarchical clustering of all 11 samples based on FPKM of all genes (Fig. 2) displayed that these samples were clustered into two branches, which indicated that the most significant change of transcriptome happened between 24 and $42 \mathrm{~h}$.

FDR $\leq 0.05$ was considered as the differential expression between samples of two time points in this study. By comparison, the DEGs between transcriptome of each two phases were identified (Additional file 2: Figure S1). A total of 515 DEGs were collected as a union set after the combination of all DEGs from two-time point comparison. Most DEGs appeared between 24 and $42 \mathrm{~h}$ during the continuous process, which is consistent with the result of clustering. And the least DEGs appeared between middle and late phases of oxygen limitation.

To determine the function of these DEGs, the 515 DEGs were then analyzed by gene ontology (GO) annotation and KEGG enrichment analysis (Additional file 3: Figure S2). Results showed that most DEGs were enriched in biological process (BP) ontology of regulation

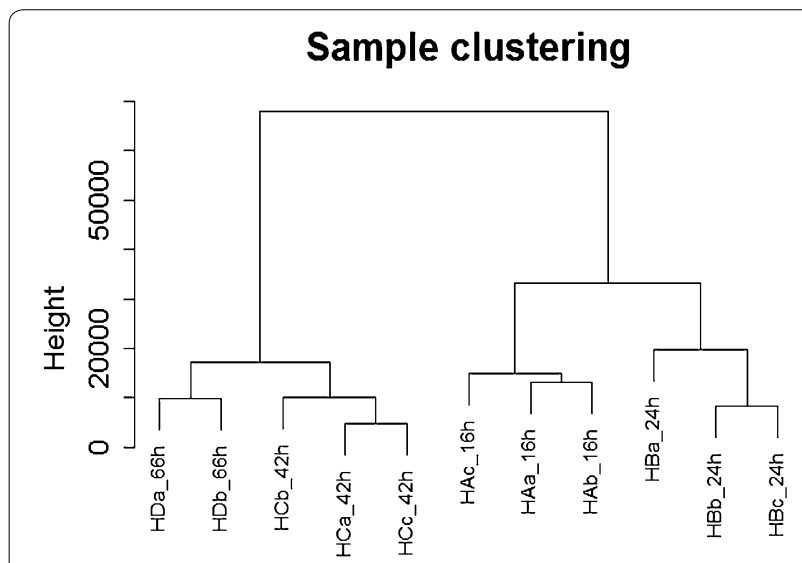

Fig. 2 Hierarchical clustering plot for all transcriptome samples of biological process, transport, RNA metabolic process, response to stress, lipid metabolic process, and molecular function (MF) ontology of hydrolase activity, oxidoreductase activity, transferase activity, and so on. As for KEGG pathways, the most DEGs were enriched in translation (40 genes), followed by the carbohydrate metabolism (35 genes), amino acid metabolism (29 genes), and lipid metabolism (23 genes).

In order to research the changes in gene expression during the glucoamylase fermentation, the expression pattern of all 515 DEGs was illustrated via heat map (Fig. 3a) and the hierarchical clustering analysis was performed using unweighted pair group method with arithmetic mean (Fig. 3b). Each profile represented a class of statistical trend of gene expression in continuous 4 time points. Then all DEGs were clustered into 12 expression patterns. We put the most concern in $\mathrm{c} 1, \mathrm{c} 2$, and c9, c10 which may represent the direct response to the continuous decrease of DO and all genes inside c1, c2 were down-regulated, and all genes within c9, c10 were up-regulated during the whole fermentation process (Fig. 3b).

According to GO and KEGG analysis of these two expression patterns (Table 2), overrepresented Go terms in Profiles $\mathrm{c} 1$ and $\mathrm{c} 2$ containing 171 down-regulated genes mainly belong to translation such as ribosome, tRNA synthase, ribonucleoprotein complex, aminoacyltRNA biosynthesis, tRNA aminoacylation. This was also consistent with the result in previous part.

Profiles c9, c10 (Table 2) containing 221 up-regulated genes with a lower significance of enrichment were compared to profiles c1, c2. Genes involved in fatty acid degradation, synthesis of aspartate and proline, a variety of hydrolase, transporter, and organic acid synthesis were up-regulated. The gene glaA encoding glucoamylase was continuously up-regulated (Fig. 4), ensuring the efficient biosynthesis of glucoamylase in this strain.

\section{Transcriptomic response of metabolic pathways} during glucoamylase fermentation

From the iPath map based on the comparison of transcriptomic data of 24 and $42 \mathrm{~h}$, fatty acid metabolism 


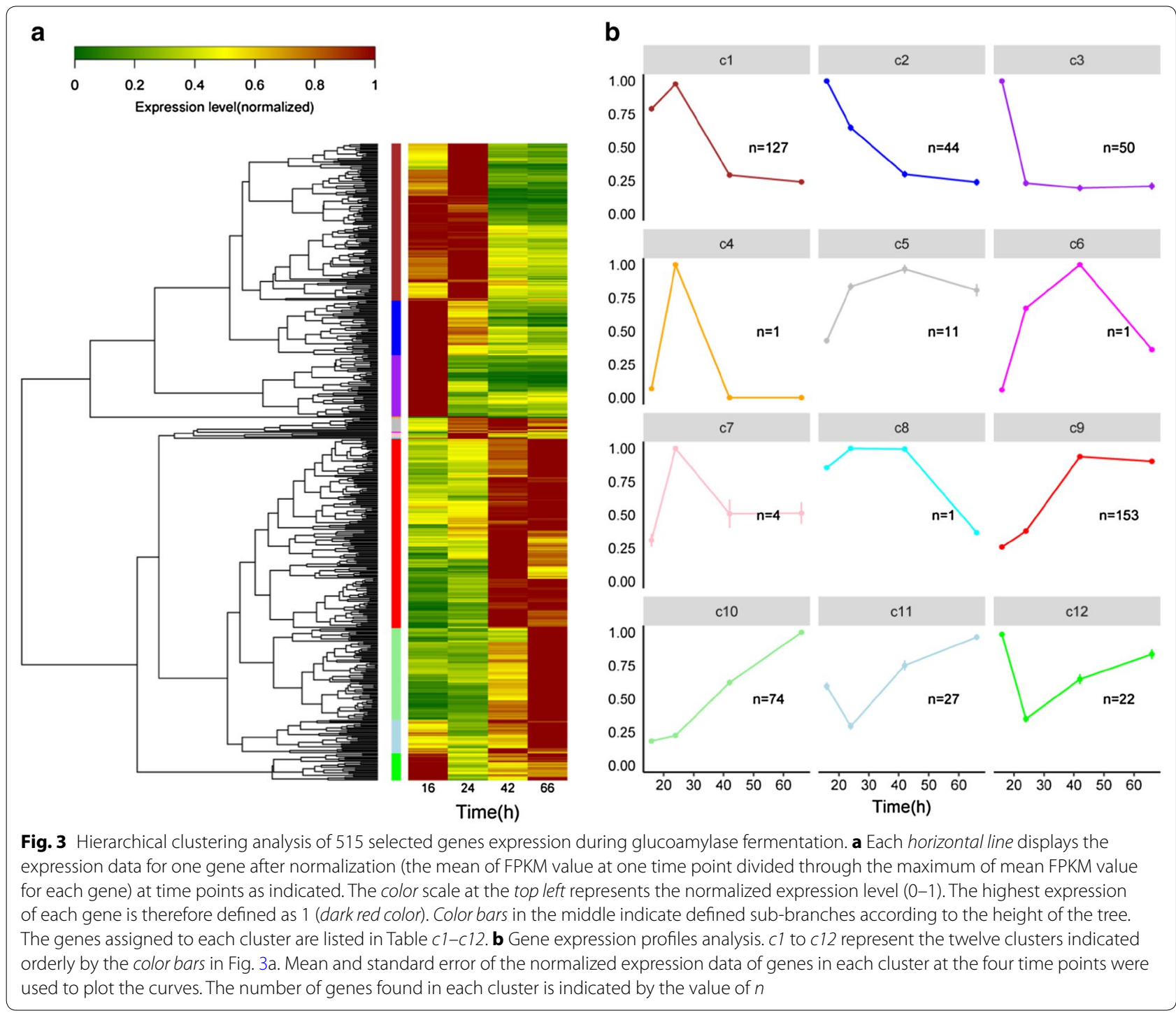

pathways were up-regulated significantly. Moreover, the most significant down-regulated pathway was fatty acid biosynthesis (Additional file 4: Figure S3). The oxidative phosphorylation pathway was also significantly reduced, while the fatty acid catabolism was strongly enhanced. Considering the slowdown of biomass synthesis, energy generated from enhanced fatty acid catabolism may flow mostly to the biosynthesis of glucoamylase, leading to the dramatic increase of enzyme yield on biomass.

Referred to the amino acid composition of glucoamylase (Table 3), Ser, Thr, and Ala take up more than $10 \%$ of the total amino acids, respectively. In addition, Leu, Gly, Asp, and Val are also the main compositions as the proportion of each of them is more than 6\%. Among them, Thr takes Asn as its precursor and Ala, Leu, and Val can be transformed from pyruvate. It is worth to pay attention that the expression levels of pyruvate kinase (An07g08990) and asparagine synthase (An04g01340) were very high, also showing the trend of up-regulation during the whole fermentation process. Although the expression level of pyruvate kinase was decreased at $24 \mathrm{~h}$ compared to that at $16 \mathrm{~h}$, it then increased to the same level as $16 \mathrm{~h}$. The high expression level and up-regulation of pyruvate kinase and asparagine synthase ensured the high concentration of pyruvate and oxaloacetic acid as the carbon skeleton of Thr, Ala, Leu, and Val, which is consistent with the previous literature that the intermediates of TCA cycle were accumulated under the oxygen limitation (Diano et al. 2009). What's more, 3-phosphoglyceric acid as the carbon skeleton of Ser and Gly, the high expression level of its biosynthesis gene An11g01390 (glycerolate dehydrogenase) guaranteed the biosynthesis efficiency of serine and glycine. Except for these six 
Table 2 Gene ontology (GO) enrichment analysis of four overrepresented clusters c1, c2 and c9, c10

\begin{tabular}{|c|c|c|c|c|}
\hline GO term & Description GO term & Count & Ontology & $p$ value \\
\hline \multicolumn{5}{|c|}{ Profiles c1, c2 (down-regulated) } \\
\hline GO:0005840 & Ribosome & 11 & $\mathrm{CC}$ & $1.13 \mathrm{E}-07$ \\
\hline GO:0030529 & Ribonucleoprotein complex & 11 & $\mathrm{CC}$ & $9.56 \mathrm{E}-07$ \\
\hline GO:0003735 & Structural constituent of ribosome & 11 & MF & $1.57 \mathrm{E}-06$ \\
\hline GO:0006412 & Translation & 14 & $\mathrm{BP}$ & $2.29 \mathrm{E}-06$ \\
\hline GO:0005198 & Structural molecule activity & 11 & MF & $8.26 \mathrm{E}-06$ \\
\hline GO:0043228 & Non-membrane-bounded organelle & 12 & $\mathrm{CC}$ & $1.03 E-05$ \\
\hline GO:0043232 & Intracellular non-membrane-bounded & 12 & $\mathrm{CC}$ & $1.03 \mathrm{E}-05$ \\
\hline GO:0008654 & Phospholipid biosynthetic process & 3 & $\mathrm{BP}$ & 0.059 \\
\hline GO:0006399 & tRNA metabolic process & 4 & $\mathrm{BP}$ & 0.055 \\
\hline GO:0043038 & Amino acid activation & 3 & $\mathrm{BP}$ & 0.088 \\
\hline GO:0043039 & tRNA aminoacylation & 3 & $\mathrm{BP}$ & 0.088 \\
\hline \multicolumn{5}{|c|}{ Profiles c9, c10 (up-regulated) } \\
\hline GO:0008080 & $\mathrm{N}$-acetyltransferase activity & 3 & MF & 0.19 \\
\hline GO:0016410 & $\mathrm{N}$-acyltransferase activity & 3 & MF & 0.20 \\
\hline GO:0016407 & Acetyltransferase activity & 3 & MF & 0.27 \\
\hline GO:0044271 & Nitrogen compound biosynthetic process & 8 & $\mathrm{BP}$ & 0.099 \\
\hline GO:0008652 & Cellular amino acid biosynthetic process & 3 & $\mathrm{BP}$ & 0.41 \\
\hline GO:0009309 & Amine biosynthetic process & 3 & $\mathrm{BP}$ & 0.43 \\
\hline GO:0016053 & Organic acid biosynthetic process & 3 & $\mathrm{BP}$ & 0.51 \\
\hline GO:0046394 & Carboxylic acid biosynthetic process & 3 & $\mathrm{BP}$ & 0.51 \\
\hline GO:0000166 & Nucleotide binding & 23 & MF & 0.21 \\
\hline GO:0032555 & Purine ribonucleotide binding & 16 & MF & 0.29 \\
\hline GO:0032553 & Ribonucleotide binding & 16 & MF & 0.29 \\
\hline GO:0017076 & Purine nucleotide binding & 18 & MF & 0.36 \\
\hline GO:0005524 & ATP binding & 13 & MF & 0.43 \\
\hline GO:0032559 & Adenyl ribonucleotide binding & 13 & MF & 0.43 \\
\hline GO:0001883 & Purine nucleoside binding & 15 & MF & 0.50 \\
\hline GO:0030554 & Adenyl nucleotide binding & 15 & MF & 0.50 \\
\hline GO:0001882 & Nucleoside binding & 15 & MF & 0.51 \\
\hline GO:0015399 & Primary active transmembrane transporter activity & 3 & MF & 0.44 \\
\hline GO:0005694 & Chromosome & 3 & CC & 0.19 \\
\hline GO:0003677 & DNA binding & 15 & MF & 0.39 \\
\hline GO:0030528 & Transcription regulator activity & 11 & MF & 0.42 \\
\hline
\end{tabular}

amino acids, the gene expression level of the biosynthesis pathways of other amino acids was relatively low.

To compare with the published transcriptomic study on carbon starvation, we specially analyzed the expression profile of genes related to carbohydrate active enzymes (CAZymes), hydrolases (chitinase, glucanase, protease, and phosphatidase), transporter, and the formation of conidiophore, which responded strongly under carbon starvation. Unlike carbon starvation, the genes related to CAZymes and formation of conidiophore did not respond in a wide and strong way in this study.

A wider range response of genes encoding hydrolase and oxidoreductase were induced under oxygen-limited phase, but the tendencies are obscure. As for transporter
(865 in total) (Pel et al. 2007), 29 genes were expressed significantly differently and most of them (21 genes) were up-regulated, indicating an intense increase of transporter activity.

\section{Transcriptional changes relevant to secretion pathway}

As an important expression vector of various homogenous and heterogenous proteins, the protein secretion pathway of $A$. niger gained much attention by researchers (Kwon et al. 2012; Jørgensen et al. 2009; Carvalho et al. 2012; Guillemette et al. 2007). By summarizing data of these published literature, a total of 465 genes involved in secretion pathways of $A$. niger were collected. In all of the 515 DEGs, about 23 DEGs (12 up-regulated genes 


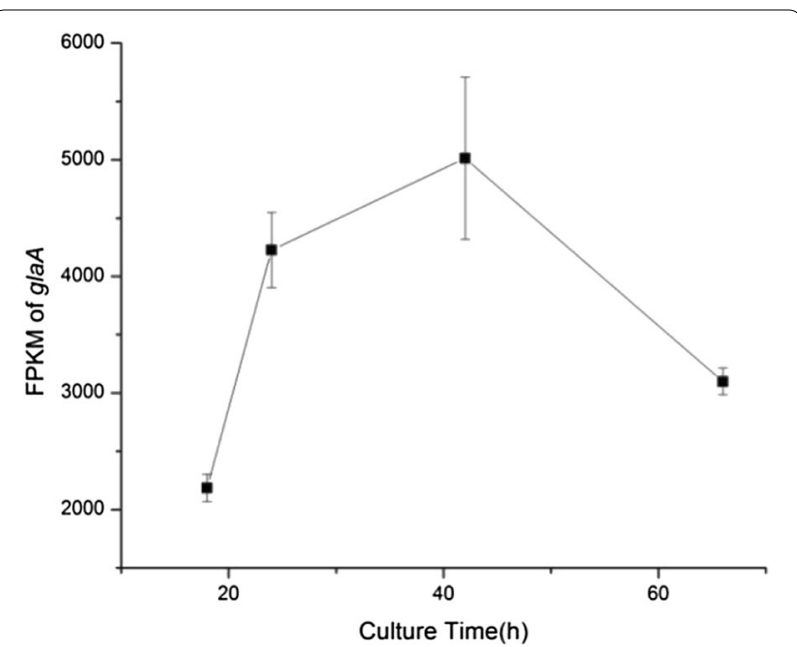

Fig. 4 Gene expression of glaA encoding glucoamylase during fermentation. All FPKM of genes were calculated based on triplicate biological samples and the error bar means the error among three biological replicate samples

Table 3 The amino acid composition of glucoamylase

\begin{tabular}{llcc}
\hline Amino acid(s) & Number count & \% by weight & \% by frequency \\
\hline A Ala & 65 & 7.24 & 10.14 \\
C Cys & 10 & 1.52 & 1.56 \\
D Asp & 44 & 7.33 & 6.86 \\
E Glu & 26 & 4.78 & 4.06 \\
F Phe & 22 & 4.55 & 3.43 \\
G Gly & 47 & 4.41 & 7.33 \\
H His & 4 & 0.78 & 0.62 \\
I lle & 24 & 3.94 & 3.74 \\
K Lys & 13 & 2.38 & 2.03 \\
L Leu & 48 & 7.88 & 7.49 \\
M Met & 3 & 0.56 & 0.47 \\
N Asn & 25 & 4.13 & 3.90 \\
P Pro & 22 & 3.17 & 3.43 \\
Q Gln & 17 & 3.11 & 2.65 \\
R Arg & 20 & 4.36 & 3.12 \\
S Ser & 88 & 11.57 & 13.73 \\
T Thr & 74 & 11.03 & 11.54 \\
V Val & 42 & 6.15 & 6.55 \\
W Trp & 19 & 4.85 & 2.96 \\
Y Tyr & 27 & 6.12 & 4.21 \\
\hline
\end{tabular}

in cluster 8,9,10,11,12, and 11 down-regulated genes in cluster 1,2) were distributed in ten secretory pathways, including protein folding, ER-associated degradation (ERAD), protein complex involved in protein transport, proteins involved in vesicle formation and docking, ER to Golgi and intra-Golgi transport, genes and transcription factors related to iron ion absorption, protease biosynthesis, and glycosylation processes.

$B i p A$ encoding the main chaperone protein in the endoplasmic reticulum (ER) was up-regulated before $24 \mathrm{~h}$ and then down-regulated, possibly because that the specific productivity of glucoamylase reached the peak at $24 \mathrm{~h}$ and then decreased. It is also a critical gene on unfolded protein response (UPR) pathway (Maattanen et al. 2010), and is beneficial to promote the correct folding of proteins in ER; thus, its down-regulated expression in this study possibly shows that endoplasmic reticulum stress response was not reduced in the process of glucoamylase fermentation. Three DEGs (An04g06180, An02g01690, An08g10650) as components of COPII-coated vesicles were enriched in protein complex involved in gene protein transport pathway. COPII-coated vesicle-mediated protein transports from ER to Golgi, and therefore, it was predicted that up-regulated genes involved in the components of COPII-coated vesicles may contribute to the transport of glucoamylase. Two genes related to starch metabolism: aamA (An11g03340) encoding acid amylase and glaA (An03g06550) encoding glucoamylase were both significantly up-regulated. Among genes encoding extracellular protease, An02g13410, encoding predicted acetyl coenzyme A transporter, is involved in the transport of membrane permeability COA from the cytoplasm to the inner membrane of ER for transient acetylating resident proteins on ER, which can improve the folding efficiency of secretory protein (Kwon et al. 2012). Compared with the data in literature, oxygen limitation did not cause the wide range response of secretory pathways during glucoamylase production, as most of the genes on secretory pathways such as UPR, ERAD did not show significant differences in expression. The result indicated that secretory pathways may not act as a restrictive factor in the yield of glucoamylase in glucoamylase-producing strain A. niger DS03043.

\section{Transcriptional changes relevant to transcription factor}

Fungal transcription factor database (FTFD, http://ftfd. snu.ac.kr/intro.php) (Park et al. 2008), known as currently the most complete $A$. niger transcription factor database, included 775 transcription factors of $A$. niger. Combining the data of the literature (Jørgensen et al. 2009), FTFD database (Park et al. 2008) and STRING database (protein-protein interaction network) (Punt et al. 2008), a total of 869 transcription factors (TFs) in our transcriptomic data were identified. Among them, $4 \mathrm{TFs}$, viz. srbB (An14g02540), prtT (An04g06940), An11g07350, and An17g01370 were significantly up-regulated during fermentation (Table 4). The expression level of $s r b B$ and $p r t T$ in the middle stage of oxygen limitation reached 2-3 times higher than that in the exponential growth 
phase, but the most significant difference in TF expression was An17g01370, as the expression level of which at $42 \mathrm{~h}$ was more than 10 times of that at $16 \mathrm{~h}$. According to the NCBI annotation, An17g01370 is related to DNA repair, containing $\mathrm{Zn}$-binding and two AraC-type DNAbinding domains, but the role of this TF in $A$. niger has not been reported yet. PrtT, belonging to the class of the Zn (II) 2Cys6-binuclear cluster family, is the only identified specific protease transcription factor in A. niger, which not only regulates the expression of many extracellular proteases, but also enhances the expression of genes involved in iron uptake (Hagag et al. 2012). Punt et al. obtained a $\operatorname{prtT}$ mutant by random mutagenesis, resulting in a significant decrease of protease activity (Punt et al. 2008). The homolog of prtT in Aspergillus oryzae regulates the major alkaline protease AlpA and the neutral protease Np1. Sharon (Sharon et al. 2009) knocked out the homologous gene of the prtT in Aspergillus fumigatus, resulting in the loss of secretory protease activity, in which six secretory proteases were significantly reduced (ALP, MEP, Dpp4, CpdS, AFUA_2G17330, and AFUA_7G06220). In our transcriptome data, several genes encoding common extracellular proteases such as $p e p A$, pepB, and pepE displayed the trend of up-regulation (but not belonging to DEGs), predicting the increase of the biosynthesis of extracellular proteases during the fermentation, which may result in the degradation of glucoamylase and not conducive to the accumulation of glucoamylase. Therefore, it is presumed that the yield of glucoamylase could be increased further by downregulating the expression of some protease genes or transcription factor PrtT to decrease the activity of protease.

Sterol-regulatory element-binding proteins (SREBP) transcription factor SrbA belonging to the basic HelixLoop-Helix (bHLH) family plays an important role in the resistance to antifungal drugs and in the virulence of Aspergillus fumigatus. Chung et al. (2014) verified the genes directly bound by $s r b A$ in $A$. fumigatus by ChIPseq approach. They confirmed that SrbA not only directly regulates the synthesis of sterols and the uptake of iron, but also is a global transcription factor under hypoxia conditions. Its biological roles include ensuring the normal growth of hyphae, regulating mycelium polarization, assimilating nitrate, changing components of cell wall through the regulation of sterol biosynthesis pathways, regulating the virulence of pathogens, and regulating biosynthesis of amino acids, fatty acids, and lipid. SrbB, whose sequence is very similar to SrbA, co-regulates genes involved in heme biosynthesis and demethylation of C4-sterols with SrbA in hypoxia condition in $A$. fumigatus. In addition, SrbB has regulatory functions independent of SrbA including regulation of carbohydrate metabolism. In our transcriptome data, both $\operatorname{srb} A$ and $\operatorname{srb} B$ showed an up-regulated trend during fermentation, but the expression level of $s r b A$ was constantly low, while $\operatorname{srb} B$ expressed in a high level from the beginning and increased dramatically during middle and late oxygen limitation phase, indicating SrbB has important roles in the transcriptional regulation in hypoxia fermentation of $A$. niger. Thirty genes directly regulated by SrbA in A. fumigatus were obtained by ChIP-seq approach. All the 30 target genes of $s r b A$ have homologous genes in $A$. niger genome. However, when searching for genes containing the same SrbA binding motif ( $5^{\prime}-(\mathrm{A} / \mathrm{G})$ TCA $\left.(\mathrm{T} / \mathrm{C} / \mathrm{G})(\mathrm{C} / \mathrm{G}) \mathrm{CCAC}(\mathrm{T} / \mathrm{C})-3^{\prime}\right)$ as reported in $A$. fumigatus in $A$. niger's genome, the matching rate was low, indicating the binding motif of SrbA in $A$. niger may be very different from that of $A$. fumigatus. Moreover, the expression of most enzymes in sterol biosynthesis pathways was relatively low. The possible explanation is that

Table 4 Summary of important up-regulated transcription factors in the process of glucoamylase fermentation

\begin{tabular}{|c|c|c|c|c|c|c|c|}
\hline \multirow[t]{2}{*}{ Gene ID } & \multirow[t]{2}{*}{ Alter name } & \multicolumn{4}{|c|}{$\begin{array}{l}\text { FPKM value of each gene } \\
\text { in the four sampling time } \\
\text { points in this study }\end{array}$} & \multirow[t]{2}{*}{ Annotation in A. niger } & \multirow[t]{2}{*}{ References } \\
\hline & & $16 \mathrm{~h}$ & $24 \mathrm{~h}$ & $42 \mathrm{~h}$ & $66 \mathrm{~h}$ & & \\
\hline An04g06940 & prtT & 267.5 & 105.1 & 335.1 & 362.5 & $\begin{array}{l}\text { Member of the fungal-specific Zn2Cys6-binuclear } \\
\text { cluster protein family; transcriptional activator of } \\
\text { major secreted proteases and also enhances the } \\
\text { expression of genes involved in iron uptake }\end{array}$ & $\begin{array}{l}\text { Punt et al. (2008); Hagag } \\
\text { et al. (2012); Sharon et al. } \\
\text { (2009) }\end{array}$ \\
\hline An14g02540 & $\operatorname{srb} B$ & 382.6 & 292.3 & 792.7 & 1095.7 & $\begin{array}{l}\text { HLH DNA-binding domain protein, sterol-regulatory } \\
\text { element-binding protein response to hypoxia; SrbB } \\
\text { co-regulates genes involved in heme biosynthesis } \\
\text { and demethylation of C4-sterols with SrbA }\end{array}$ & $\begin{array}{l}\text { Cerqueira et al. (2014); Park } \\
\text { et al. (2008); Chung et al. } \\
\text { (2014) }\end{array}$ \\
\hline An11g07350 & & 885.3 & 1635.8 & 407.6 & 267.4 & $\begin{array}{l}\text { Ortholog(s) have role in positive regulation of sec- } \\
\text { ondary metabolite biosynthetic process; } \\
\text { Function- GABA utilization is amdR-dependent in } A \text {. } \\
\text { oryzae ( } 777 \text { aa) }\end{array}$ & $\begin{array}{l}\text { Cerqueira et al. (2014); Park } \\
\text { et al. (2008) }\end{array}$ \\
\hline An17g01370 & Ada & 26.3 & 95.8 & 326.9 & 306.6 & DNA repair and transcription factor Ada & Punt et al. (2008) \\
\hline
\end{tabular}


as a lung pathogenic fungi, the environmental oxygen for $A$. fumigatus is very low (about only $1 \%$ ). During the glucoamylase fermentation of $A$. niger, although dissolved oxygen was relative low, the oxygen ratio was still about 20\%. The stress level was much different from the hypoxic condition as A. fumigatus faced in Chung's study.

\section{Conclusion}

In this study, we analyzed the transcriptome samples at 4 time points to study the global transcriptional response of industrial glucoamylase-producing strain $A$. niger DS03043 in the process of glucoamylase fermentation and preliminarily revealed the mechanism of adapting to oxygen limitation fermentation and efficient biosynthesis of glucoamylase. In the fermentation process, oxygen limitation caused by the increasing amount of biomass reduced the expression of genes in oxidative phosphorylation pathway, biosynthesis of proteins, and fatty acids immediately and constantly, which lead to the breakup of biomass synthesis. When the cell growth slowdown, saved energy and precursors from enhanced catabolism of fatty acids inclined to flow to the biosynthesis of products. The high gene copy of glaA ensured the high expression level of this gene in this condition. In addition, increased expression of genes related to transporter, oxidoreductase, and synthesis of asparagine and pyruvate also contribute to the high yield of glucoamylase in oxygen limitation. Genes in secretory pathways have not shown a widespread response in A. niger DS03043, indicating that secretory pathways may be not a limiting factor for the yield of glucoamylase in oxygen-limited fermentation.

In this paper, we analyzed the transcriptional profile of different stages in fermentation process referred by physiological parameters, which contributed to a better understanding of the effect of oxygen limitation fermentation on gene expression and cell metabolism. It further helps us to identify the targets useful for improving the productivity of the cell factory.

\section{Additional files}

Additional file 1: Table S1. All differentially expressed genes in the process of glucoamylase fermentation.

Additional file 2: Figure S1. iPath map of transcriptional level changes on metabolic pathways between $42 \mathrm{~h}$ and $24 \mathrm{~h}$.

Additional file 3: Figure S2. GO and KEGG function enrichment analysis of all DEGs.

Additional file 4: Figure S3. DEGs generated by two-time point comparison.

\section{Authors' contributions}

YFS was in charge of paper writing. LM directed the study as the tutor and modified the manuscript. LFL, SC, and GYZ performed the bioinformatics analysis and modified the manuscript. HN offered experimental strain and modified the manuscript. All authors read and approved the final manuscript.

\section{Author details}

${ }^{1}$ State Key Laboratory of Bioreactor Engineering, East China University of Science and Technology, Shanghai 200237, People's Republic of China.

2 BGI-Shenzhen, Shenzhen 518083, China. ${ }^{3}$ DSM Biotechnology Center, Delft, Netherlands.

Acknowledgements

Not applicable.

\section{Competing interests}

The authors declare that they have no competing interests.

\section{Availability of data and materials}

The datasets supporting the conclusions of this article are included in the main manuscript file and additional files.

Ethics approval and consent to participate and consent for publication All authors have read and approved the manuscript before submitting it to bioresources and bioprocessing. There are no competing interests to declare by any author in relation to the submission.

\section{Funding}

This work was funded by the Basic Research Program of Shenzhen (JCYJ20150629165423751) and the Open Funding Project of the State Key Laboratory of Bioreactor Engineering.

\section{Publisher's Note}

Springer Nature remains neutral with regard to jurisdictional claims in published maps and institutional affiliations.

Received: 9 May 2017 Accepted: 30 June 2017

Published online: 14 October 2017

\section{References}

Andersen MR, Salazar MP, Schaap PJ (2011) Comparative genomics of citric-acid-producing Aspergillus niger ATCC 1015 versus enzyme-producing CBS 513.88. Genome Res 21(6):885-897

Baker SE (2006) Aspergillus niger genomics: past, present and into the future. Med Mycol 44(Suppl 1):17-21

Carvalho ND, Jørgensen TR, Arentshorst M, Nitsche BM, van den Hondel CA, Archer DB, Ram AF (2012) Genome-wide expression analysis upon constitutive activation of the HacAbZIP transcription factor in Aspergillus niger reveals a coordinated cellular response to counteract ER stress. BMC Genom 13(1):105

Cerqueira GC, Arnaud MB, Inglis DO, Skrzypek MS, Binkley G, Simison M, Miyasato SR, Binkley J, Orvis J, Shah P, Wymore F, Sherlock G, Wortman JR (2014) The Aspergillus Genome Database: multispecies curation and incorporation of RNA-Seq data to improve structural gene annotations. Nucleic Acids Res 42(1):D705-D710

Chung D, Barker BM, Carey CC, Merriman B, Werner ER, Lechner BE, Dhingra S, Cheng C, Xu W, Blosser SJ, Morohashi K, Mazurie A, Mitchell TK, Haas H, Mitchell AP, Cramer RA (2014) ChIP-seq and in vivo transcriptome analyses of the Aspergillus fumigatus SREBP SrbA reveals a new regulator of the fungal hypoxia response and virulence. PLoS Pathog 10(11):e1004487

Delmas S, Pullan ST, Gaddipati S, Kokolski M, Malla S, Blythe MJ, Ibbett R, Campbell M, Liddell S, Aboobaker A, Tucker GA, Archer DB (2012) Uncovering the genome-wide transcriptional responses of the filamentous fungus Aspergillus niger to lignocellulose using RNA sequencing. PLoS Genet 8(8):e1002875

Diano A, Bekker-Jensen S, Dynesen J, Nielsen J (2006) Polyol synthesis in Aspergillus niger: influence of oxygen availability, carbon and nitrogen sources on the metabolism. Biotechnol Bioeng 94(5):899-908 
Diano A, Peeters J, Dynesen J, Nielsen J (2009) Physiology of Aspergillus niger in oxygen-limited continuous cultures: influence of aeration, carbon source concentration and dilution rate. Biotechnol Bioeng 103(5):956

Guillemette T, van Peij N, Goosen T, Lanthaler K, Robson GD, van den Hondel CA, Stam H, Archer DB (2007) Genomic analysis of the secretion stress response in the enzyme-producing cell factory Aspergillus niger. BMC Genom 8:158

Hagag S, Kubitschek-Barreira P, Neves GW, Amar D, Nierman W, Shalit I, Shamir R, Lopes-Bezerra L, Osherov N (2012) Transcriptional and proteomic analysis of the Aspergillus fumigatus $\Delta$ prtT protease-deficient mutant. PLOS ONE 7(4):e33604

Huang DW, Sherman BT, Lempicki RA (2009a) Systematic and integrative analysis of large gene lists using DAVID bioinformatics resources. Nat Protoc 4(1):44-57

Huang DW, Sherman BT, Lempicki RA (2009b) Bioinformatics enrichment tools: paths toward the comprehensive functional analysis of large gene lists. Nat Acids Res 37(1):1-13

Jørgensen TR, Goosen T, Hondel CA, Ram AF, Iversen JJ (2009) Transcriptomic comparison of Aspergillus niger growing on two different sugars reveals coordinated regulation of the secretory pathway. BMC Genom 10(1):44

Jørgensen TR, Nitsche BM, Lamers GE, Arentshorst M, van den Hondel CA, Ram AF (2010) Transcriptomic insights into the physiology of Aspergillus niger approaching a specific growth rate of zero. Appl Environ Microbiol 76(16):5344-5355

Kanehisa M, Goto S (2000) KEGG: kyoto encyclopedia of genes and genomes Nucleic Acids Res 28:27-30

Kanehisa M, Sato Y, Kawashima M, Furumichi M, Tanabe M (2016) KEGG as a reference resource for gene and protein annotation. Nucleic Acids Res 44:D457-D462

Kwon MJ, Jørgensen TR, Nitsche BM, Arentshorst M, Park J, Ram AF, Meyer V (2012) The transcriptomic fingerprint of glucoamylase over-expression in Aspergillus niger. BMC Genom 13:701

Li B, Dewey CN (2011) RSEM: accurate transcript quantification from RNA-Seq data with or without a reference genome. BMC Bioinform 12:323

Lu H, Liu X, Huang M, Xia J, Chu J, Zhuang Y, Zhang S, Noorman H (2015) Integrated isotope-assisted metabolomics and $13 \mathrm{C}$ metabolic flux analysis reveals metabolic flux redistribution for high glucoamylase production by Aspergillus niger. Microb Cell Fact 14:147

Maattanen P, Gehring K, Bergeron JJ, Thomas DY (2010) Protein quality control in the ER: the recognition of misfolded proteins. Semin Cell Dev Biol 21(5):500-511

Ning L, Dawson JA, Thomson JA, Ruotti V, Rissman Al, Smits BMG, Haag JD, Gould MN, Stewart RM, Kendziorski C (2013) EBSeq: an empirical Bayes hierarchical model for inference in RNA-Seq experiments. Bioinformatics 29(8):1035-1043
Nitsche BM, Jørgensen TR, Akeroyd M, Meyer V, Ram AF (2012) The carbon starvation response of Aspergillus niger during submerged cultivation: insights from the transcriptome and secretome. BMC Genom 13:380

Novodvorska M, Hayer K, Pullan ST, Wilson R, Blythe MJ, Stam H, Stratford M, Archer DB (2013) Transcriptional landscape of Aspergillus niger at breaking of conidial dormancy revealed by RNA-sequencing. BMC Genom 194(1):246

Park J, Park J, Jang S, Kim S, Kong S, Choi J, Ahn K, Kim J, Lee S, Kim S, Park B, Jung K, Kim S, Kang S, Lee YH (2008) FTFD: an informatics pipeline supporting phylogenomic analysis of fungal transcription factors. Bioinformatics 24(7):1024-1025

Pavezzi FC, Carneiro AA, Bocchini-Martins DA, Alves-Prado HF, Ferreira H, Martins PM, Gomes E, da Silva R (2011) Influence of different substrates on the production of a mutant thermostable glucoamylase in submerged fermentation. Appl Biochem Biotechnol 163(1):14-24

Pedersen H, Beyer M, Nielsen J (2000) Glucoamylase production in batch, chemostat and fed-batch cultivations by an industrial strain of Aspergillus niger. Appl Microbiol Biotechnol 53(3):272-277

Pedersen L, Hansen K, Nielsen J, Lantz AE, Thykaer J (2012) Industrial glucoamylase fed-batch benefits from oxygen limitation and high osmolarity. Biotechnol Bioeng 109(1):116-124

Pel HJ, de Winde JH, Archer DB et al (2007) Genome sequencing and analysis of the versatile cell factory Aspergillus niger CBS 513.88. Nat Biotechnol 25(2):221-231

Punt PJ, Schuren FH, Lehmbeck J, Christensen T, Hjort C, van den Hondel CA (2008) Characterization of the Aspergillus niger prtT, a unique regulator of extracellular protease encoding genes. Fungal Genet Biol 45:1591-1599

Sharon H, Hagag S, Osherov N (2009) Transcription factor PrtT controls expression of multiple secreted proteases in the human pathogenic mold Aspergillus fumigatus. Infect Immun 77(9):4051-4060

Shi SB, Chen T, Zhao XM (2010) Transcriptome platforms and application to metabolic engineering. Chin J Biotech 26(9):1187-1198 (in Chinese)

van Leeuwen MR, Krijgsheld P, Wyatt TT, Golovina EA, Menke H, Dekker A, Stark J, Stam H, Bleichrodt R, Wösten HAB, Dijksterhuis J (2012) The effect of natamycin on the transcriptome of conidia of Aspergillus niger. Stud Mycol 74(1):71-85

van Munster JM, Daly P, Delmas S, Pullan ST, Blythe MJ, Malla S, Kokolski M, Noltorp EC, Wennberg K, Fetherston R, Beniston R, Yu X, Dupree P, Archer DB (2014) The role of carbon starvation in the induction of enzymes that degrade plant-derived carbohydrates in Aspergillus niger. Fungal Genet Biol 72:34-47

Yamada T, Letunic I, Okuda S, Kanehisa M, Bork P (2011) iPath2.0: interactive pathway explorer. Nucleic Acids Res 39(suppl 2):W412-W415

\section{Submit your manuscript to a SpringerOpen ${ }^{\circ}$ journal and benefit from:}

- Convenient online submission

- Rigorous peer review

- Open access: articles freely available online

- High visibility within the field

Retaining the copyright to your article

Submit your next manuscript at springeropen.com 